\title{
Application of circuit simulation method for differential modeling of TIM-2 iron uptake and metabolism in mouse kidney cells
}

\author{
Zhijian Xie ${ }^{1}$, Scott H. Harrison ${ }^{2}$, Suzy V. Torti ${ }^{3}$, Frank M. Torti ${ }^{4}$ and Jian Han ${ }^{2 *}$ \\ ${ }^{1}$ Department of Electrical Engineering, North Carolina Agricultural and Technical State University, Greensboro, NC, USA \\ 2 Department of Biology, North Carolina Agricultural and Technical State University, Greensboro, NC, USA \\ ${ }^{3}$ Department of Molecular, Microbial, and Structural Biology, University of Connecticut Health Center, Farmington, CT, USA \\ ${ }^{4}$ Department of Medicine, School of Medicine, University of Connecticut Health Center, Farmington, CT, USA
}

\section{Edited by:}

Xiaogang Wu, Indiana

University-Purdue University

Indianapolis, USA

\section{Reviewed by:}

Jongrae Kim, University of Glasgow,

UK

Guanglong Jiang, Capital Normal University, China

Zhiping Wang, Indiana University, USA

\section{*Correspondence:}

Jian Han, Department of Biology, North Carolina Agricultural and

Technical State University, 1601 E Market St, Greensboro, NC 27411, USA

e-mail: jhan@ncat.edu
Circuit simulation is a powerful methodology to generate differential mathematical models. Due to its highly accurate modeling capability, circuit simulation can be used to investigate interactions between the parts and processes of a cellular system. Circuit simulation has become a core technology for the field of electrical engineering, but its application in biology has not yet been fully realized. As a case study for evaluating the more advanced features of a circuit simulation tool called Advanced Design System (ADS), we collected and modeled laboratory data for iron metabolism in mouse kidney cells for a $\mathrm{H}$ ferritin (HFt) receptor, T cell immunoglobulin and mucin domain-2 (TIM-2). The internal controlling parameters of TIM-2 associated iron metabolism were extracted and the ratios of iron movement among cellular compartments were quantified by ADS. The differential model processed by circuit simulation demonstrated a capability to identify variables and predict outcomes that could not be readily measured by in vitro experiments. For example, an initial rate of uptake of iron-loaded HFt (Fe-HFt) was 2.17 pmol per million cells. TIM-2 binding probability with Fe-HFt was $16.6 \%$. An average of $8.5 \mathrm{~min}$ was required for the complex of TIM-2 and Fe-HFt to form an endosome. The endosome containing HFt lasted roughly $2 \mathrm{~h}$. At the end of endocytosis, about $28 \% \mathrm{HFt}$ remained intact and the rest was degraded. Iron released from degraded HFt was in the labile iron pool (LIP) and stimulated the generation of endogenous HFt for new storage. Both experimental data and the model showed that TIM-2 was not involved in the process of iron export. The extracted internal controlling parameters successfully captured the complexity of TIM-2 pathway and the use of circuit simulation-based modeling across a wider range of cellular systems is the next step for validating the significance and utility of this method.

Keywords: circuit simulator, export, ferritin, iron, model, storage, TIM-2, uptake

\section{BACKGROUND}

There have been both classical and advanced uses of analogous electronic circuit concepts in evaluating biological systems. For more than several decades, both animal and plant physiologists have used models such as Ohm's Law to model environmental response (Janes, 1970; Meier et al., 2003). A modern challenge has been to discover and interrelate cellular dynamics with higher-level outcomes (Kitano, 2002a). Biochemical systems theory (BST) provides a conceptual foundation for differential analysis of the functional requirements and design principles of a viable cell (Savageau, 1972, 1979, 2001). Electrical circuits are also subject to differential analysis of their linear and nonlinear components (McAdams and Shapiro, 1995). We propose that circuit

Abbreviations: ADS, Advanced Design System; DC, direct current; FeNTA, ferrous nitrotriacetic acid; Fpn, ferroportin; LIP, labile iron pool; HFt, H ferritin; ODE, ordinary differential equations; PBS, phosphate buffered saline; SDS, sodium dodecyl sulphate; SSNE, sum of squares due to normalized error; TIM-2, $\mathrm{T}$ cell immunoglobulin and mucin domain-2; Tf, transferrin; TfR 1, Transferrin receptor 1 . simulation may be a powerful technique for realizing the potential of BST within the $21^{\text {st }}$ century discipline of computational systems biology, a field that aspires to evaluate complex biological systems through the use of computers (Kitano, 2002b).

Circuit simulation software has been extensively developed by semiconductor and electronics industries to handle circuit topologies having complex objectives for optimization and having many diverse interconnected components. Circuit simulation for biological systems was attempted several decades ago in the early years of the digital age (Thomas and Mikulecky, 1978), but usage has been infrequent. Its relevance may be renewed now both by a strong community effort to extensively crowd source the computer modeling of cells (Helikar et al., 2012), and by transformative developments such as a whole cell simulation of phenotype that illustrate the prowess of drawing together a wide range of mathematical models for cellular genome expression (Karr et al., 2012). After new experimental findings go beyond the original knowledge for the modeled system, there is a need to model the newly discovered subsystem and integrate it into the 
prior model. Contemporary circuit simulation software provides an agile platform for inputting a differential model and extending it with a rich feature set of advanced numerical and optimization methods.

To test a circuit simulation approach, we sought to examine iron, a cellular micronutrient for which both modeling and a new wave of experimental data exists. Outside of cells, sources of iron in the bloodstream include both transferrin (Tf) and ferritin (Ft). There are complex multicellular conditions of disease associated with increased serum levels of iron-loaded $\mathrm{Tf}$ and iron-loaded $\mathrm{Ft}$ (Konijn et al., 1981; Arad et al., 1988; Torti and Torti, 1994), and the underlying mechanisms and regulatory effects for cell-serum iron transport are complex and variable across different species (Kuchroo et al., 2003; Knickelbein et al., 2006; Rennert et al., 2006; Watanabe et al., 2007; Todorich et al., 2008; Rodriguez-Manzanet et al., 2009; Rejniak et al., 2010). Recently, a mouse-specific T cell immunoglobulin and mucin domain containing (TIM) protein receptor, $\mathrm{T}$ cell immunoglobulin and mucin domain-2 (TIM2 ), has been found to process iron delivery. Regulatory effects of TIM-2 have been identified in both mouse brain glial cells (Watanabe et al., 2007) and kidney cells (Han et al., 2011). Han et al. (2011) found that TIM-2 uptakes iron from exogenous $\mathrm{H}$ ferritin $(\mathrm{HFt})$. Ferritin is an iron storage and delivery protein made of both $\mathrm{H}$ and $\mathrm{L}$ subunits (Han et al., 2000; Todorich et al., 2011).

Although a mathematical model of iron metabolism in mammalian cells has been recently proposed (Chifman et al., 2012), this model does not account for the uptake of iron by TIM-2. It is based upon iron uptake through the classical transferrintransferrin receptor pathway and storage of iron within ferritin (Klausner et al., 1983; Baynes et al., 1987). State variables account for the movement of iron between a labile iron pool (LIP) and four types of proteins - transferrin receptor 1 (TfR1), exporter ferroportin (Fpn), HFt, and active iron regulatory proteins (IRPs) (Chifman et al., 2012). In this study, we extended this model by developing governing equations for an additional set of TIM-2 dynamics and comparing outcomes of a differential model-based circuit simulation to laboratory data. Laboratory data were collected in vitro for different times of exposure of HFt to TIM-2 cDNA transfected mouse kidney TCMK-1 cells. The outcome of the comparison between simulation data and laboratory data showed circuit simulation to be a valuable tool for quantifying emerging knowledge of the integral and complex role that micronutrients have in cellular physiology.

\section{MATERIALS AND METHODS IRON UPTAKE AND IRON STORAGE DATA}

Data and the experimental procedures for iron uptake and iron storage analysis are from (Han et al., 2011). Brief summaries of iron uptake and storage methods are described below.

\section{Iron uptake study}

$125 \mu \mathrm{Ci}^{55} \mathrm{FeCl}_{3}$ was added to $50 \mu \mathrm{g} / \mathrm{ml}$ mouse recombinant $\mathrm{HFt}$ in buffer of $20 \mu \mathrm{M}$ citric acid, $2 \mathrm{mM}$ ascorbate, and 0.1 M HEPES (pH 6.0) (Santambrogio et al., 1993). ${ }^{55} \mathrm{Fe}-\mathrm{HFt}$ complex was then filtered through a $0.45 \mu \mathrm{m}$ syringe filter. Two $\mu \mathrm{g} / \mathrm{ml}{ }^{55} \mathrm{Fe}-\mathrm{HFt}$ complex was added to $1 \times 10^{6}$ TCMK- 1 vector or TIM- 2 cells and the cells were incubated at $37^{\circ} \mathrm{C}$ for $0,5,15,30,60,90$, and $120 \mathrm{~min}$. Cells were washed three times with PBS and harvested in whole cell lysis buffer [ $25 \mathrm{mM}$ Tris $\mathrm{pH} 7.4,1 \%$ Triton $\mathrm{X}-100,1 \%$ sodium dodecyl sulfate (SDS), 1\% sodium deoxycholate, $150 \mathrm{mM} \mathrm{NaCl}, 2 \mu \mathrm{g} / \mathrm{ml}$ aprotinin, $1 \mathrm{mM}$ PMSF, complete protease inhibitor (Roche Diagnostics, Indianapolis, IN)]. The radio-activity of ${ }^{55} \mathrm{Fe}$ was measured. The experiment was performed in triplicate.

\section{Iron storage experiment}

$125 \mu \mathrm{Ci}{ }^{55} \mathrm{Fe}$ was loaded into dialyzed biotinylated HFt and then dialyzed in $0.1 \mathrm{M}$ HEPES at $4^{\circ} \mathrm{C}$. TCMK- 1 TIM- 2 or vector cells were incubated with $2 \mu \mathrm{g} / \mathrm{ml}$ biotinylated $-{ }^{55} \mathrm{Fe}-\mathrm{HFt}$ at $37^{\circ} \mathrm{C}$ for $2 \mathrm{~h}$. Plates were washed with PBS and placed in PC-1 growth media. Collection times were at $0,2,4,8$, 24 , and $48 \mathrm{~h}$ after incubation. Cell lysates were prepared by homogenization in lysis buffer for $5 \mathrm{~s}$, and then centrifuged at $12,000 \times \mathrm{g}$ at $4{ }^{\circ} \mathrm{C}$ for $15 \mathrm{~min}$. Biotin $-{ }^{55} \mathrm{Fe}-\mathrm{HFt}$ was immunoprecipitated from the supernatant by incubation with streptavidin conjugated beads (Jackson Immuno Research, PA) (Wang et al., 2007). The beads were dissolved in $10 \% \mathrm{SDS} / 0.1 \mathrm{M}$ $\mathrm{NaOH}$ solution and the ${ }^{55} \mathrm{Fe}$ radio-activity was measured. The radio-activity of ${ }^{55} \mathrm{Fe}$ from extracts depleted of biotinylated ferritin was also measured. The experiment was performed in triplicate.

\section{CHEMICALS AND CELL CULTURES FOR IRON EXPORT EXPERIMENT}

Chemicals and cell cultures were purchased and handled as previously described (Han et al., 2011). The TCMK-1 mouse kidney epithelial cell line was obtained from the American Type Culture Collection (ATCC, Rockville, MD). Transfection of TIM-2 was performed with vector plasmids [BSR- $\alpha$-FLAG (Chen et al., 2005)]. Selection for stable transfectants of TIM-2 was as previously described (Han et al., 2011).

\section{IRON EXPORT EXPERIMENT}

To test if TIM-2 can be the exporter of HFt and iron, TCMK1 vector and TIM-2 containing cells were pre-loaded with ${ }^{55} \mathrm{Fe}$ Tf. The biotin-labeled apo-HFt was added to the cells. Biotin-HFt and ${ }^{55} \mathrm{Fe}$ in media were examined as exported products.

\section{Iron labeling of mouse transferrin (Tf)}

$125 \mu \mathrm{Ci}{ }^{55} \mathrm{FeCl}_{3}$ was mixed with $10 \mu \mathrm{l}$ of $10 \mathrm{mM}$ NTA ( $\mathrm{pH}$ was adjusted to 6 using $1 \mathrm{M} \mathrm{NaHCO} 3$ ). The mixture was incubated with $2 \mathrm{mg} / \mathrm{ml}$ mouse apo-Tf in $0.2 \mathrm{M} \mathrm{NaOAc}$ at room temperature for $30 \mathrm{~min}$. The rest of Tf binding sites were saturated with $100 \mu \mathrm{l}$ of $10 \mathrm{mM}$ cold FeNTA at room temperature for $30 \mathrm{~min}$. The product was dialyzed in $0.02 \mathrm{M}$ Tris- $\mathrm{HCl}(\mathrm{pH}=7)$ at $4^{\circ} \mathrm{C}$ overnight and the absorbance at A465 and A280 was measured $(\mathrm{A} 465 / \mathrm{A} 280=0.057$, near to the ideal reading of 0.045$)$ (Baynes et al., 1987).

\section{Iron preloading and apo-HFt treatment to the cells}

TCMK-1 vector and TIM-2 containing cells were incubated with $0.37 \mu \mathrm{M} \mathrm{Tf}-{ }^{55} \mathrm{Fe}$ for $4 \mathrm{~h}$ at $37^{\circ} \mathrm{C}$ in culture containing $5 \% \mathrm{CO}_{2}$. Cells were then incubated with $2 \mu \mathrm{g} / \mathrm{ml}$ biotin labeled apo-HFt for $3 \mathrm{~h}$. Cells were washed with PBS and changed into normal 
growth media containing 10\% FBS. Media were collected at 0, 2, 4, 24, and $48 \mathrm{~h}$. ${ }^{55} \mathrm{Fe}$ in media was considered exported product and measured by liquid scintillation counter.

\section{Pulling down biotin-labeled HFt in the media using streptavidin beads}

Media at different time points were each incubated with streptavidin conjugated beads at $4^{\circ} \mathrm{C}$ overnight. Beads were spun down at $4000 \mathrm{rpm}$ for $5 \mathrm{~min}$ and then dissolved in $10 \% \mathrm{SDS} / 0.1 \mathrm{M}$ $\mathrm{NaOH}$ solution and incubated at $50^{\circ} \mathrm{C}$ for $1 \mathrm{~h}$. Activity of ${ }^{55} \mathrm{Fe}$ was counted from streptavidin pulling down solution. The experiment was performed in triplicate.

\section{GOVERNING EOUATIONS OF IRON METABOLISM}

A mathematical model of iron homeostasis developed by Chifman et al. (2012) was used as a basis for proposing an extended model with the TIM-2 pathway. Governing equations based upon this model for time derivatives (without the TIM-2 pathway) are described in Equations 1-5 with state variables $x_{1}=[\mathrm{LIP}], x_{2}=$ [TfR 1$], x_{3}=[\mathrm{Fpn}], x_{4}=[\mathrm{HFt}]$, and $x_{5}=$ [Active IRPs].

$$
\begin{aligned}
& \dot{x}_{1}=\alpha_{1} F e_{e x} x_{2}+\gamma_{4} x_{4}-\alpha_{6} x_{1} x_{3}-\alpha_{4} x_{1} \frac{k_{54}}{k_{54}+x_{5}} \\
& \dot{x}_{2}=\alpha_{2} \frac{x_{5}}{k_{52}+x_{5}}-\gamma_{2} x_{2} \\
& \dot{x}_{3}=\alpha_{3} \frac{k_{53}}{k_{53}+x_{5}}-\left(\gamma_{3}+\gamma_{h} H e p\right) x_{3} \\
& \dot{x}_{4}=\alpha_{4} x_{1} \frac{k_{54}}{k_{54}+x_{5}}-\gamma_{4} x_{4} \\
& \dot{x}_{5}=\alpha_{5} \frac{k_{15}}{k_{15}+x_{1}}-\gamma_{5} x_{5}
\end{aligned}
$$

Independent self-degrading behaviors for each state variable are captured by the terms $-\gamma_{i} x_{i}$, where $\gamma_{i}$ are decay constants. For effects of state variable $x_{i}$ on state variable $x_{j}$, terms in the form of $\frac{\alpha_{j} x_{i}}{k_{i j}+x_{i}}$ represent the promoting effect, and terms in the form of $\frac{\alpha_{j} k_{i j}}{k_{i j}+x_{i}}$ represent the inhibiting effect. Activation thresholds are represented by $k_{i j}$, and maximum production rates are represented by $\alpha_{j}$. In Equation 1, second-order reaction rates are represented by $\alpha_{1} F e_{e x} x_{2}$ and $-\alpha_{6} x_{1} x_{3}$ for iron uptake and iron export, respectively. The remaining term of Equation $1,-\dot{x}_{4}$, models the dynamic where, as ferritin degrades, iron is released and elevates the level of the LIP.

The model was extended with a pathway of exogenous ironloaded HFt (Fe-HFt) as shown in Figure 1. Four dynamic states of TIM-2 were modeled: $x_{6}=$ [TIM- 2 active on cell membrane], $x_{7}=$ [TIM-2 bounded with HFt], $x_{8}=$ [TIM-2 in endosomes], and $x_{9}=$ [exogenous Ft in cell]. Time derivatives for the governing equations of this model are described in Equations 6-9.

$$
\begin{aligned}
& \dot{x}_{6}=\gamma_{6} x_{8}-\alpha_{7} F t_{e x} x_{6} \\
& \dot{x}_{7}=\alpha_{7} F t_{e x} x_{6}-\gamma_{7} x_{7} \frac{k_{47}}{k_{47}+\left(x_{4}+x_{9}\right)} \\
& \dot{x}_{8}=\gamma_{7} x_{7} \frac{k_{47}}{k_{47}+\left(x_{4}+x_{9}\right)}-\gamma_{6} x_{8} \\
& \dot{x}_{9}=\alpha_{9} \gamma_{7} x_{7}-\gamma_{9} x_{9}
\end{aligned}
$$

The term $\alpha_{7} F t_{\text {ex }} x_{6}$ is the combination rate of active TIM- 2 with exogenous ferritin, where $\alpha_{7}$ is the reaction-rate constant and $F t_{e x}$ the concentration of exogenous ferritin. The reaction turns the active TIM-2 into a bounded one, thus this term is negative in the $\dot{x}_{\epsilon}$ equation, and positive in the $\dot{x}_{7}$ equation. The concentration of

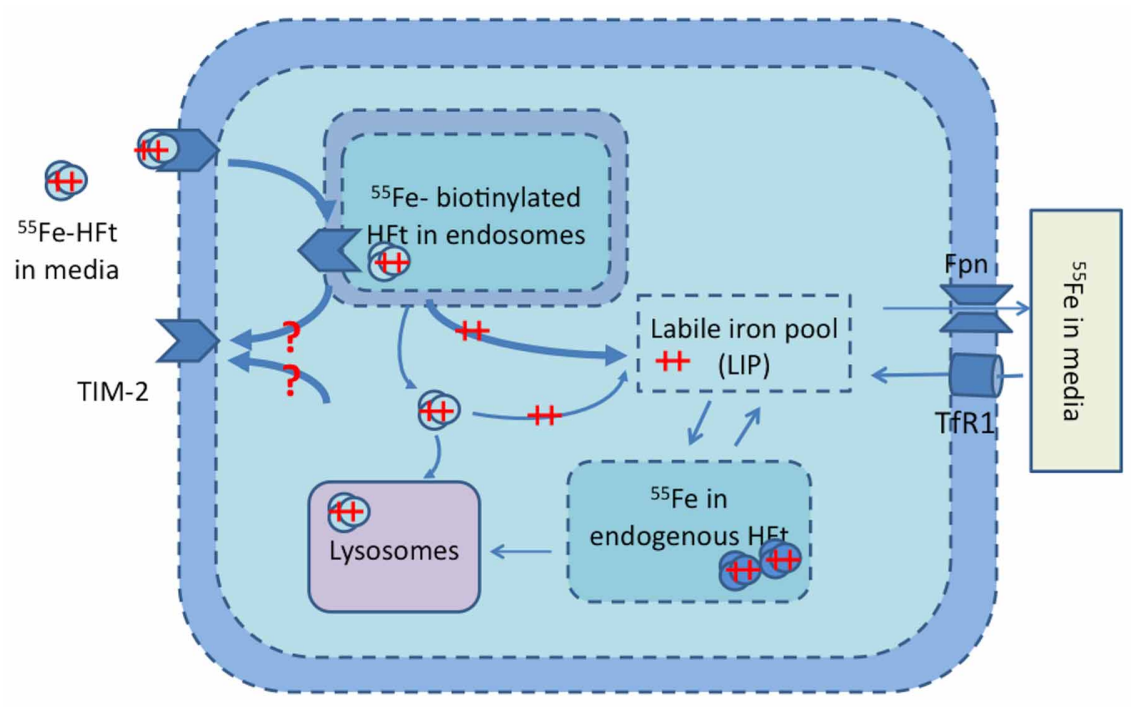

FIGURE 1 | TIM-2 pathway model of the TIM-2 receptor, endosome formation, HFt degradation, and iron release into the iron labile pool. Iron is subsequently either stored in endogenous HFt or exported to the media. Iron uptake by the TIM-2 receptor occurs at the cellular membrane.
++ represents Fe (ferrous state), and ++ with ovals represents Fe-HFt. Unshaded ovals are for external HFt, and shaded ovals are for endogenous HFt. The question marks (?) represent pathways that do not have any direct experimental support. 
TIM-2 active on cell membrane is not only affected by the combination reaction with exogenous ferritin, but also by the process of recycling them back to cell membrane. The rate of recycling is assumed to be equal to the rate of decrease of TIM-2 in the endosome. The rate of decrease of TIM-2 is modeled by a first-order decay with a decay constant $\gamma_{6}$.

The endocytosis of TIM-2 and the bounding of TIM-2 on the membrane with $\mathrm{H}$-ferritin are hindered by the presence of ferritin and are expressed in Equations 7 and 8. $\gamma_{7}$ is the rate for endocytosis with low concentration of ferritin $\left(x_{4}+x_{9}\right)$. The endosome formation saturation factor $K_{47}$ is the threshold value of ferritin concentration when the endocytosis rate is reduced by half. Equation 9 expresses the surviving exogenous iron loaded ferritin after the endosome is dissolved and its decay rate in normal cell solution. The Equation 1 time derivative of ${ }^{55} \mathrm{Fe}$ in LIP is therefore modified as shown in Equation 10. Equations 2 through 10 describe therefore the TIM-2 iron uptake and metabolism model.

$$
\begin{aligned}
\dot{x}_{1}= & \alpha_{1} F e_{e x} x_{2}+\gamma_{4} x_{4}-\alpha_{6} x_{1} x_{3} \\
& -\alpha_{4} x_{1} \frac{k_{54}}{k_{54}+x_{5}}+\alpha_{10} \gamma_{7} x_{7}+\alpha_{11} \gamma_{9} x_{9}
\end{aligned}
$$

\section{SIMULATION}

Simulation relied upon the quantified activity of ${ }^{55} \mathrm{Fe}$ where concentrations from in vitro experiments are inferred from the strength of radiation. Assumptions were that the ratios of concentration to strength of radiation were each constant for various iron-loaded proteins. Other assumptions were that initial conditions were related to the preparation process, and that the system reaches equilibrium states before and after external experimental conditions (treatments) perturbed the system. For simplicity, this work assumed that all three iron metabolic processes (uptake, storage, and export) are in equilibrium and the level of iron concentration is below the nonlinear threshold. For this simulation, the nonlinear effects which are not related to TIM-2 are ignored. Output parameters to be compared with experimental measurements were the sum of different iron-containing components.

The ordinary differential equations (ODEs) for the TIM-2 pathway of iron uptake and metabolism were mapped to an equivalent electrical circuit to be implemented within a circuit simulator. The circuit simulator used in this work was Agilent Advanced Design System (Agilent ADS). Electric circuit components were mapped to the variables and equations of the TIM-2 pathway model (Figure 2). A unit capacitor was used to hold the state variables, $x_{i}=Q_{i}=V_{i}$. Time derivative variables were represented by the current flow in and out of a capacitor, $\frac{d x_{i}}{d t}=I$. Dual directional processes were represented by a resistor connecting two capacitors, $\frac{d x_{i}}{d t}=-\frac{\left(x_{i}-x_{j}\right)}{R}$ and $\frac{d x_{j}}{d t}=\frac{\left(x_{i}-x_{j}\right)}{R}$. Unidirectional processes were represented by a current-control current source, $\frac{d x_{i}}{d t}=-\frac{x_{i}}{R_{1}}$ and, $\frac{d x_{i}}{d t}=\frac{x_{i}}{R_{2}}$. Nonlinear relations can be implemented by nonlinear-equation based sub-circuit blocks. Figure 3A shows an example of a linear to saturation model $\frac{\alpha_{j} x_{i}}{k_{i j}+x_{i}}$ term and Figure 3B shows an example of a constant to suppression model $\frac{\alpha_{j} k_{i j}}{k_{i j}+x_{i}}$ term.

A simulation "bench" was constructed with Agilent ADS as shown in Figure 4 (Agilent ADS manual, http://www.agilent. com, Santa Clara, CA, USA). With proper conversion, mathematical equations can be mapped to equivalent circuits and solved through transient simulation for dynamic process or direct current (DC) simulation for stable states. Conversion consists of three parts: mathematical equation conversion, initial condition conversion, and output parameter conversion. Controlling parameters were extracted from in vitro experiments of iron uptake, storage and export as were performed in TCMK-1 vector

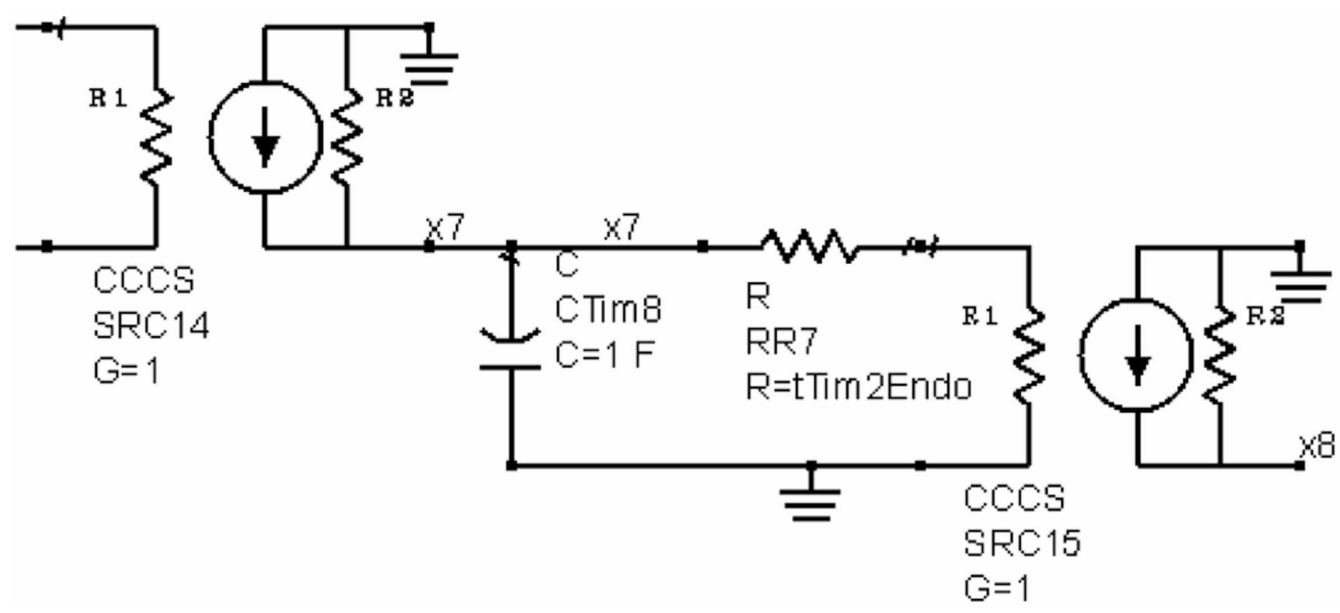

FIGURE 2 | An example circuit generated by ODE-to-circuit conversion. The component on the left is a current-control current source (CCCS), an ideal element for current scaling. The scaling factor $\mathrm{G}=1$ for the element mirrors the current, representing iron atoms in a TIM-2/ferritin complex. The current inputs into a capacitor are to model the state of iron accumulation. At a linear release condition, the stored charge in capacitor leaks through the resistor $\mathrm{RR7}$, modeling the release of iron. The current is then mirrored again on the right side of the circuit to input into another state representing iron atoms in a TIM-2/ferritin complex. 

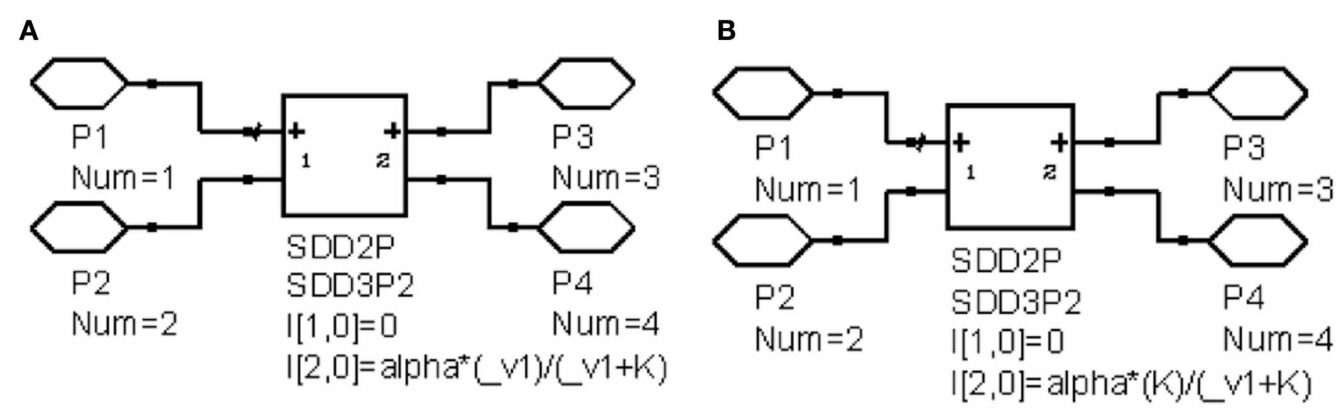

FIGURE 3 | Example circuits of nonlinear behavior. (A) Linear to saturation model; (B) Constant to suppression model.

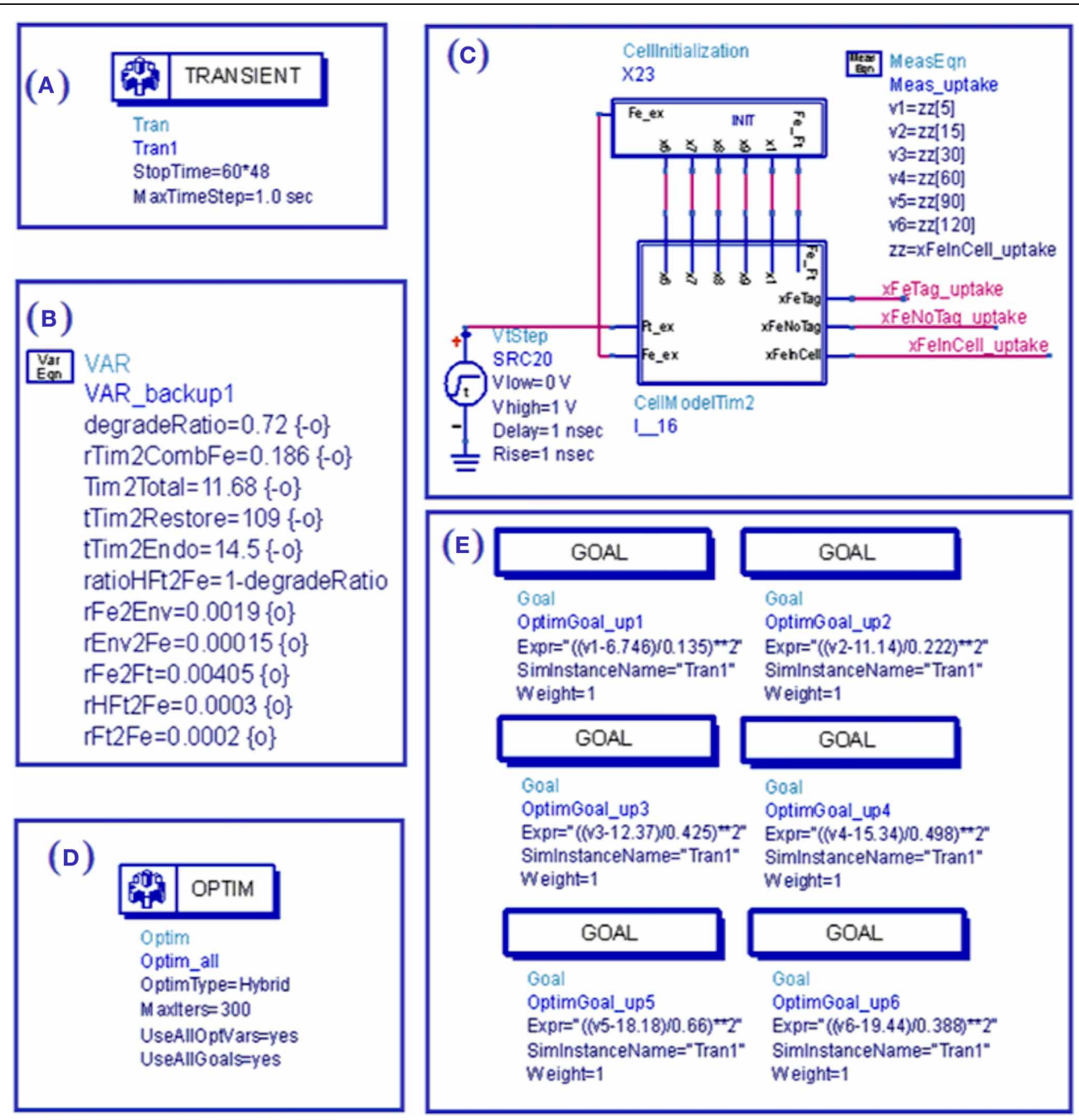

FIGURE 4 | View of ADS simulation bench for simulation of TIM-2 pathway model. (A) Simulation setup defines the time duration and resolution; (B) Variable setup defines parameters and their range for optimization or tuning; (C) Initial condition and environment (external source) as applied to the cell subcircuit through wire connections; (D) Optimization setup of optimization methods and iteration time; (E) Goals for optimization. and TIM-2 containing cells. Although the experiments were performed independently, the underlying mechanisms for iron metabolism would be the same since the same cell line was used. Therefore, the model with the same internal controlling parameters should be able to describe the underlying mechanisms for these three experiments. The goal of optimization for the overall model is to find a set of internal controlling parameters that will minimize error which is modeled by the sum of squares due 
to normalized error (SSNE) as shown in Equation 11. $N$ is the total number of data points collected from three in vitro experiments. For each point $j, y_{j}$ represents the mean, $\sigma_{j}$ represents the standard deviation, and $m_{j}$ represents the modeling data. The means and standard deviations are determined from repetitions in each point.

$$
S S N E=\sum_{j=1}^{N} \frac{\left(m_{j}-y_{j}\right)^{2}}{\sigma_{j}^{2}}
$$

\section{RESULTS}

In this study, multiple TIM-2 associated iron metabolic processes: iron uptake, storage, and export were modeled simultaneously based on a direct implementation from the Agilent ADS circuit simulator software. Internal controlling parameters for TIM-2 iron pathway were extracted by ADS based on in vitro data collected from mice kidney TCMK-1 TIM-2 and vector cells. The model demonstrated a capability to identify variables and predict outcomes that could not be readily measured by in vitro experiments.

\section{IRON UPTAKE}

Circuit simulation of iron uptake accurately modeled an increase in a time-dependent manner in TIM-2 transfectants, but not for vector controls (Figure 5). While the optimization of internal controlling parameters is conducted in conjunction with other experiments, the primary parameters most affected by the data of this experiment were the concentration of ${ }^{55} \mathrm{Fe}$-loaded ferritin in the cell culture $\left(F t_{e x}\right)$, the combination coefficient $\left(\alpha_{7}\right)$, endosome forming rate $\left(\gamma_{7}\right)$, and TIM-2 recovering rate $\left(\gamma_{6}\right)$. The iron uptake rate starts at $1.4 \mathrm{pmol}$ per min per million cells, then reduces to about $0.07 \mathrm{pmol}$ per min per million cells. The initial rising (within $10 \mathrm{~min}$ ) of iron concentration is due to the combination of TIM-2 in cell membranes with Fe-HFt. The turning point at around $10 \mathrm{~min}$ indicates the saturation of the TIM-2 combining process, i.e., the number of available TIM-2 is largely reduced. The uptake curve is not flattened out; instead it keeps rising at a slower rate. This indicates the appearance of a new TIM-2 unit on the cell membranes, which may occur from either TIM-2 recycling or new synthesis.

\section{IRON STORAGE}

Circuit simulation of iron storage was consistent with iron storage occurring by the release of iron from exogenous (biotinylated) ferritin to a cellular fraction. Release of iron occurred concomitantly with degradation of biotinylated ferritin, consistent with processing through the lysosome, and in parallel with an increase in endogenous ferritin (Han et al., 2011). This dynamic and the kinetics of increase in endogenous ferritin were similar to those of degradation of ferritin in the lysosome (Radisky and Kaplan, 1998) and an empirical model established in (Han et al., 2011).

Degradation of biotinylated ferritin consisted of two phases with different degradation time constants (Figure 6). Phase one was for the first $4 \mathrm{~h}$ and phase two was for the remaining experimental period. In phases one and two, the concentration of biotinylated ferritin decreased with rates of about $20 \%$ per $\mathrm{h}$ and
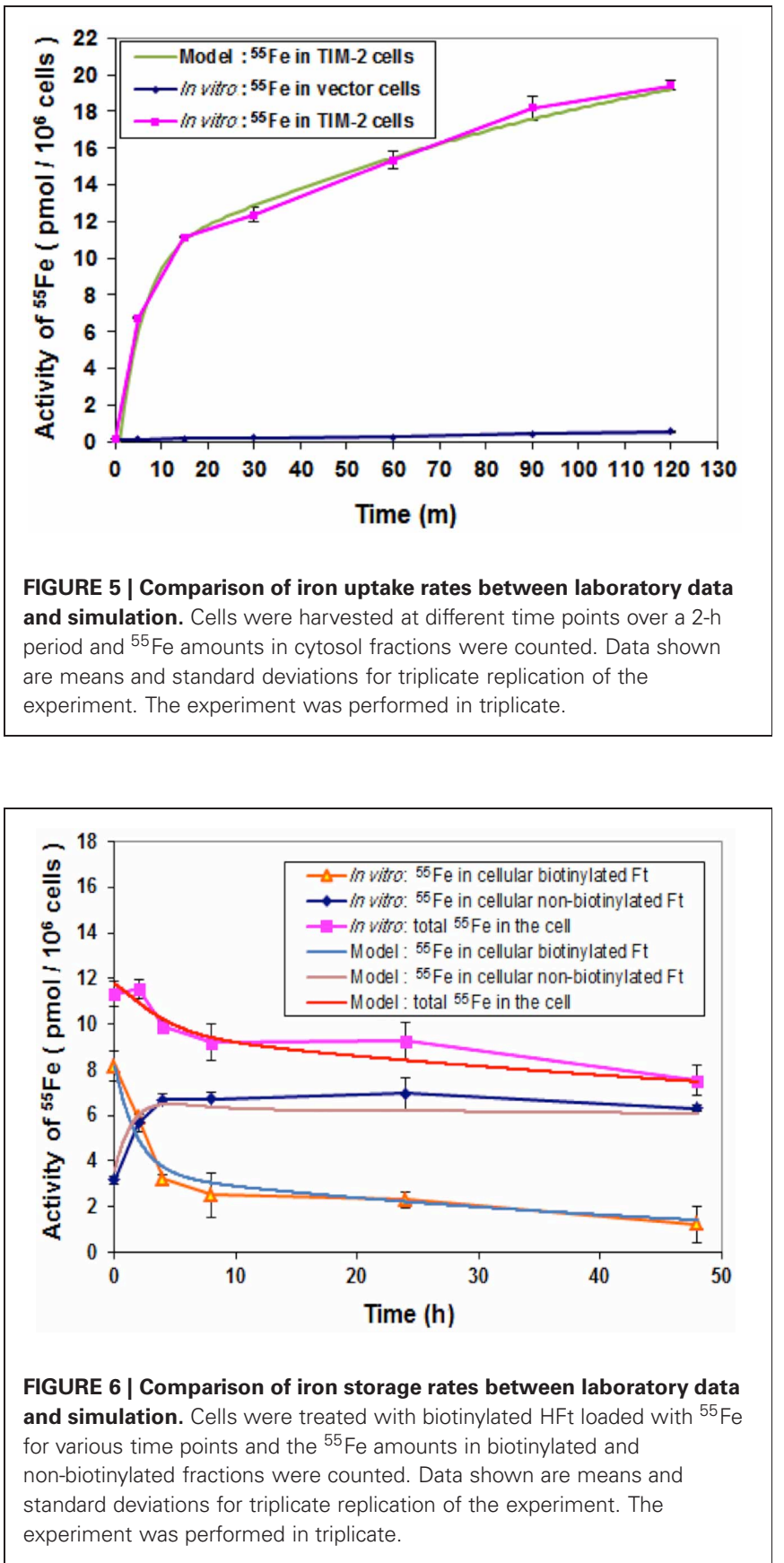

1-2\% per h, respectively. Possibly because biotinylated ferritin stayed within endosomes for approximately $2 \mathrm{~h}$ within phase one, it decreased faster due to the lower $\mathrm{pH}$ of endosome, which facilitates the ferritin degradation process in phase one. This indicates that not all of the exogenous ferritin degrades at the end of endosome (within the $2 \mathrm{~h}$ period) and the remaining ferritin continues degrading at a slower rate in the intracellular environment.

\section{IRON EXPORT}

An iron export experiment was performed for the purpose of data collection and subsequent modeling. ${ }^{55} \mathrm{Fe}$ was loaded to TCMK-1 


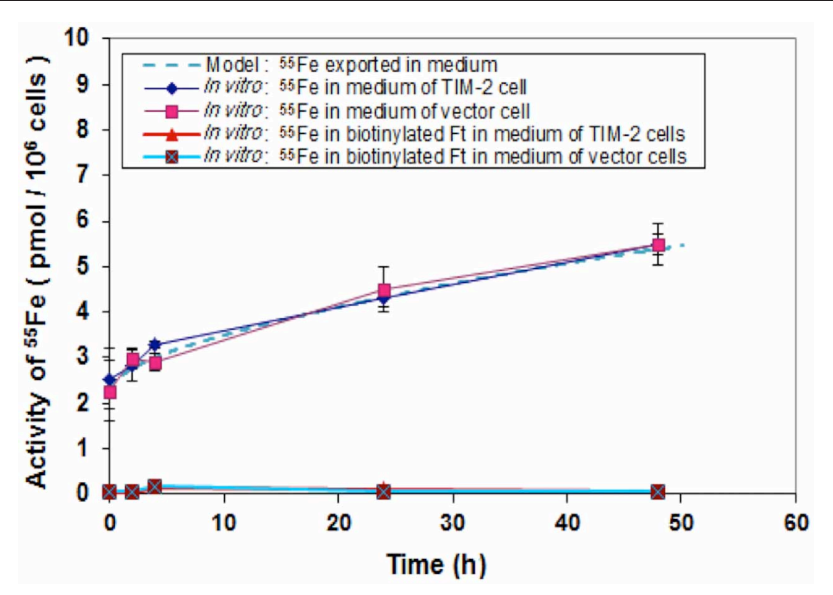

FIGURE 7 | Comparison of iron export rates between laboratory data and simulation. Cells that had been preloaded with $\mathrm{Tf}_{-}-{ }^{55} \mathrm{Fe}$ were washed and changed into normal growth media. Media were collected at different time points over a $48 \mathrm{~h}$ period and ${ }^{55} \mathrm{Fe}$ amounts in media were counted. Data shown are means and standard deviations for triplicate replication of the experiment. The experiment was performed in triplicate.

vector and TIM-2 containing cells through Tf. Biotin labeled apoHFt was treated to the cells after iron loading. ${ }^{55} \mathrm{Fe}$ and biotin labeled HFt in fresh media were measured at different time points as the exported products. The hypothesis for this study was that intracellular iron could be loaded into apo-HFt and then exported through TIM-2 receptor to the media. Our data showed that there was a general absence of biotinylated HFt in media for TCMK1 vector and TIM-2 containing cells, and essentially all of ${ }^{55} \mathrm{Fe}$ was found in total media. Figure 7 showed no difference between the amount of ${ }^{55} \mathrm{Fe}$ measured in total media of TIM- 2 and vector cells. This indicates that TIM-2 does not play a major role in iron export, so there is not a need for a separate export model for TIM2 cells. For modeling, the iron export experiments would not be expected to have an effect on determining internal controlling parameters since TIM-2 was not involved in export process.

In order to confirm that the model with extracted parameters can successfully predict the outcome of the iron export experiments, simulation runs with known initial iron concentrations in the media and the cells were conducted. With the initial iron concentration of $2.4 \mathrm{pmol}$ per million cells in the media and 10.8 pmol per million cells in the cell, the model predicted the experimental results within one standard deviation, starting with initial conditions within the experimental uncertainty. The additional information extracted from the model is that, for the iron present in the cells, $80 \%$ were stored in ferritin and 20\% is in LIP, and the export rate of ${ }^{55} \mathrm{Fe}$ from LIP is $0.2 \%$ per min for both TIM-2 and vector cells.

\section{EXTRACTED PARAMETERS}

The extracted parameters from TIM-2 models are listed in Table 1, and provide a reference set of values for TIM-2 iron metabolism pathway kinetics where partial iron is released from exogenous ferritin and stored in endogenous ferritin. The iron uptake values were consistent with the reappearance of TIM-2 at
Table 1 | Extracted controlling parameters of TIM-2 pathway model.

\begin{tabular}{|c|c|c|}
\hline $\begin{array}{l}\text { Extracted controlling } \\
\text { parameter }\end{array}$ & Expression & Value \\
\hline TIM-2 and HFt combination rate & $\alpha_{7} F t_{e x}$ & 0.166 per min \\
\hline $\begin{array}{l}\text { Initial TIM-2 endosome } \\
\text { formation rate }\end{array}$ & $\gamma_{7}$ & 0.118 per min \\
\hline $\begin{array}{l}\text { TIM-2 endosome degradation } \\
\text { rate }\end{array}$ & $\gamma_{6}$ & 0.0142 per min \\
\hline $\begin{array}{l}\text { Remaining fraction of HFt at the } \\
\text { end of endocytosis }\end{array}$ & $\alpha_{9}$ & 0.283 (unitless) \\
\hline HFt degradation rate in cell & $\gamma_{9}$ & 0.00031 per min \\
\hline $\begin{array}{l}\text { Saturation factor of endosome } \\
\text { formation }\end{array}$ & $K_{47}$ & $0.11 \mathrm{pmol}$ per million cells \\
\hline Direct iron uptake rate & $\alpha_{1} F e_{e x}$ & 0.00015 per min \\
\hline $\begin{array}{l}\text { Iron export rate through } \\
\text { ferroportin }\end{array}$ & $\alpha_{4}$ & 0.0019 per min \\
\hline
\end{tabular}

the cell surface to maintain an iron uptake flow. The extracted parameter, $\gamma_{6}=0.0142$ per $\mathrm{min}$, indicates the rate of recovery of TIM-2 after one uptake cycle. From model simulation, the recycling rate matched the consequence of the end of the fast degradation (in endosome) of the biotinylated HFt. This indicates that TIM-2 is recycled back to cell membrane. The rate of ferritin degradation was based on ${ }^{55} \mathrm{Fe}$ measurement and detection of biotinylated HFt. The two phases of ferritin degradation indicate that amounts of iron released from the ferritin molecules were different between the beginning and the end of the $48 \mathrm{~h}$ experimental period. Excess iron may have been exported through TIM-2 independent pathway, which is predictably through Fpn, a known iron exporter.

\section{DISCUSSION}

Differential modeling with circuit simulation evaluated the kinetic processes of iron relocation across three phases: iron uptake, iron storage, and iron export. Each process was represented by governing equations. The model calculated the ratios of iron movement among different cellular compartments and confirmed the specific uptake of iron via HFt through the TIM2 receptor. By using concentration values measured in vitro and applying the method of circuit simulation, precisely quantified outcomes were predicted. Specifically, controlling parameters, the ratios of uptake, storage, and export, and the recycling rate of the TIM-2 receptor were predicted and consistent with the dynamic role of the TIM-2 receptor based on iron kinetics. The continuous distribution of outcomes predicted by the simulation will ultimately, when applied to multiple host/cell lineages and environment controls, allow for focused comparisons that evaluate differences between species and physiologic conditions that impact pathways of cellular iron metabolism.

The circuit simulator simulated multiple biological processes: iron uptake, storage, and export simultaneously based on a direct implementation from the Agilent ADS circuit simulator software interface. Although the underlying processing of differential models by a circuit simulator makes itself comparable to a general performance capability of Matlab (another common tool for 
modeling biological systems), the Agilent ADS circuit simulator interface avoids a complicated coding process. When compared to other circuit simulators such as PSPICE, Agilent ADS provided an intuitive interface and wide range of features for multiple variable optimizations that we used for kinetic modeling. Finally, unlike software for generic mathematical modeling, circuit simulators are advantageous in having a software architecture that is scalable for a large number of ODEs, a feature that may be promising for expansive studies of reaction networks. A summary of these comparisons is provided in Table 2. An ongoing challenge in biological studies has been the resolution of analytical bottlenecks that frequently occur after the initial quantification of data. Provided that an analyst has a working understanding of linear control systems, the use of circuit simulation software when applied to kinetic modeling would be both flexible and straightforward based on the results of this case study.

Although the usage of biotechnology to generate data is accelerating, the inexact measurement of parameters in biological systems can limit the number of ODEs that would require a highthroughput analytical capacity. We expect however that as the scope of analysis moves beyond a limited set of model organisms and biochemical pathways, the circuit simulation approach would benefit multi-level analyses that go from molecules to entire ecosystems in an evolutionary context. The degree to which the high-throughput capacity and multiple goal optimization interface of circuit simulation software are needed would depend greatly on what is found upon investigating for pluralism in nature's "circuitry." In this study, the identification and analysis of a novel non-human pathway for a well-studied micronutrient such as iron represents a first step in uncovering such pluralism.

The simulation setup in this study was configured for a cosimulation of three experimental data sets, but the setup readily supports multiple combinations of experimental data sets to be evaluated separately or together. Prospective testing of this method for circuit simulation would include functional validation of model-based predictions. For instance, the significance of the extracted parameters in this study may be further pursued

Table 2 | Comparison between circuit simulator with Advance Design System and Matlab.

\begin{tabular}{|c|c|c|}
\hline & $\begin{array}{l}\text { Circuit simulation with } \\
\text { Advance Design System }\end{array}$ & Matlab \\
\hline Solving ODE & Yes & Yes \\
\hline $\begin{array}{l}\text { Solving ODE } \\
\text { system }\end{array}$ & Yes & Yes \\
\hline $\begin{array}{l}\text { Converging } \\
\text { control }\end{array}$ & $\begin{array}{l}\text { Select different solver both } \\
\text { automatically and manually }\end{array}$ & $\begin{array}{l}\text { Select different solver } \\
\text { manually }\end{array}$ \\
\hline Optimization & $\begin{array}{l}\text { Implemented directly } \\
\text { through interface }\end{array}$ & $\begin{array}{l}\text { Implemented through } \\
\text { programming }\end{array}$ \\
\hline $\begin{array}{l}\text { Multiple goal } \\
\text { optimization }\end{array}$ & $\begin{array}{l}\text { Implemented directly } \\
\text { through interface }\end{array}$ & $\begin{array}{l}\text { Implemented through } \\
\text { programming }\end{array}$ \\
\hline
\end{tabular}

ODE, ordinary differential equations. by comparison with other animal models for expressional variation with, or lack of, the TIM-2 receptor and conducting likelihood analysis for internal controlling parameters. We further anticipate that the merging together of multiple differential models would be useful for evaluating the complex, dynamic outcomes of a more extensive and biologically responsive cellular network.

The scalability and analytical feature set of circuit simulation is enormous, and compares favorably to other approaches found in computational modeling studies (Ulrich et al., 2006; Laubenbacher et al., 2009; Rejniak and McCawley, 2010; Rejniak et al., 2010, 2012; Salgado et al., 2010; Wu et al., 2010; Zhu et al., 2011). Advanced numerical methods include Jacobian matrix evaluation, step control, and convergence control, to handle huge amounts of linear and nonlinear electrical components. Optimization methods include genetic, random, gradient, minmax, random minmax, quasi-Newton, and least path. Different optimization methods can cover different situations such as the quality of initial guess, converge speed, and existence of local optimum conditions. This presents a powerful range of options for developing and extending models that are based upon ongoing research to identify new mechanisms within complex cellular networks.

A distinctive issue can often be whether the strength of a software application is to enable detailed customization of an analysis through scripting in a programming language, or to accelerate throughput and tractable project completion by pre-configured interface options. For the former approach, a general ideal is to enable a wide variety of approaches to connect data to analytical computations. For the latter approach, the variety of data-tocomputation modalities can be restrictive if the pre-configured interface has incorrect assumptions and/or does not provide a wide enough range of options to manage different contexts. It is therefore an interesting finding that the interface-based ADS tool, designed for an electrical engineering context, was effective at simulating biological system parameters. The unique feature of ADS, as well as other circuit simulators, is their history of solving linear and nonlinear ODE for complex and potentially massive circuits. This case report indicates that a complex analysis may be achieved in an interface-based circuit simulator without extensive knowledge of a programming language. This capability has potential application for future complex modeling of biological systems.

\section{AUTHOR'S CONTRIBUTION}

Zhijian Xie and Jian Han designed the modeling study. Suzy V. Torti and Frank M. Torti designed the in vitro experiments and coordinated the use of experimental materials. Jian Han performed the in vitro experiments. Zhijian Xie, Scott H. Harrison, and Jian Han conducted the analysis and wrote the manuscript. All authors read and approved the final manuscript.

\section{ACKNOWLEDGMENTS}

This study is supported in part by grants from the National Institutes of Health (R01DK71892, Suzy V. Torti; R37 DK42421 and R01 CA171101, Frank M. Torti). We thank Gregory D. Goins for his critical review of the manuscript. 


\section{REFERENCES}

Arad, I., Konijn, A. M., Linder, N., Goldstein, M., and Kaufmann, N. A. (1988). Serum ferritin levels in preterm infants after multiple blood transfusions. Am. J. Perinatol. 5, 40-43. doi: 10.1055/s-2007-999651

Baynes, R., Bukofzer, G., Bothwell, T., Bezwoda, W., and Macfarlane, B. (1987). Transferrin receptors and transferrin iron uptake by cultured human blood monocytes. Eur. J. Cell Biol. 43, 372-376.

Chen, T. T., Li, L., Chung, D. H., Allen, C. D., Torti, S. V., Torti, F. M., et al. (2005). TIM-2 is expressed on B cells and in liver and kidney and is a receptor for $\mathrm{H}$-ferritin endocytosis. J. Exp. Med. 202, 955-965. doi: 10.1084/jem.20042433

Chifman, J., Kniss, A., Neupane, P., Williams, I., Leung, B., Deng, Z., et al. (2012). The core control system of intracellular iron homeostasis: a mathematical model. J. Theor. Biol. 300, 91-99. doi: 10.1016/j.jtbi.2012.01.024

Han, J., Day, J. R., Thomson, K., Connor, J. R., and Beard, J. L. (2000). Iron deficiency alters $\mathrm{H}$ - and L-ferritin expression in rat brain. Cell Mol. Biol. (Noisy-le-grand) 46, 517-528.

Han, J., Seaman, W. E., Di, X., Wang, W., Willingham, M., Torti, F. M., et al. (2011). Iron uptake mediated by binding of $\mathrm{H}$-ferritin to the TIM-2 receptor in mouse cells. PLoS ONE 6:e23800. doi: 10.1371/journal.pone. 0023800

Helikar, T., Kowal, B., McClenathan, S., Bruckner, M., Rowley, T., Madrahimov, A., et al. (2012). The cell collective: toward an open and collaborative approach to systems biology. BMC Syst. Biol. 6:96. doi: 10.1186/1752-0509-6-96

Janes, B. E. (1970). Effect of carbon dioxide, osmotic potential of nutrient solution, and light intensity on transpiration and resistance to flow of water in pepper plants. Plant Physiol. 45, 95-103. doi: 10.1104/pp.45.1.95

Karr, J. R., Sanghvi, J. C., Macklin, D. N., Gutschow, M. V., Jacobs, J. M., Bolival, B. Jr., et al. (2012). A whole-cell computational model predicts phenotype from genotype. Cell 150, 389-401. doi: 10.1016/j.cell.2012.05.044

Kitano, H. (2002a). "Systems biology: towards systems-level understanding of biological systems," in Foundations of Systems Biology, ed H. Kitano (Cambridge, MA: MIT Press), 1-36.
Kitano, H. (2002b). Computational systems biology. Nature 420, 206-210. doi: 10.1038/nature01254

Klausner, R. D., Van Renswoude, J., Ashwell, G., Kempf, C., Schechter, A. N., Dean, A., et al. (1983). Receptor-mediated endocytosis of transferrin in K562 cells. J. Biol. Chem. 258, 4715-4724.

Knickelbein, J. E., de Souza, A. J., Tosti, R., Narayan, P., and Kane, L. P. (2006). Cutting edge: inhibition of $\mathrm{T}$ cell activation by TIM- 2 . J. Immunol. 177, 4966-4970.

Konijn, A. M., Carmel, N., Levy, R., and Hershko, C. (1981). Ferritin synthesis in inflammation. II. Mechanism of increased ferritin synthesis. Br. J. Haematol. 49, 361-370. doi: 10.1111/j.1365-2141. 1981.tb07238.x

Kuchroo, V. K., Umetsu, D. T., DeKruyff, R. H., and Freeman, G. J. (2003). The TIM gene family: emerging roles in immunity and disease. Nat. Rev. Immunol. 3, 454-462. doi: 10.1038/nril111

Laubenbacher, R., Hower, V., Jarrah, A., Torti, S. V., Shulaev, V., Mendes, P., et al. (2009). A systems biology view of cancer. Biochim. Biophys. Acta 1796, 129-139. doi: 10.1016/j.bbcan.2009.06.001

McAdams, H. H., and Shapiro, L. (1995). Circuit simulation of genetic networks. Science 269, 650-656. doi: $10.1126 /$ science.7624793

Meier, J., Wölkhammer, S., and Habler, O. (2003). The DeltaCrit System (DCS): a computer program for standardized bedside detection of critical oxygen delivery using the Deltatrac II metabolic monitor. Comput. Biol. Med. 33, 395-405. doi: 10.1016/S0010-4825(03)00015-5

Radisky, D. C., and Kaplan, J. (1998). Iron in cytosolic ferritin can be recycled through lysosomal degradation in human fibroblasts. Biochem. J. 336( $\mathrm{Pt} 1)$, 201-205.

Rejniak, K. A., and McCawley, L. J. (2010). Current trends in mathematical modeling of tumor-microenvironment interactions: a survey of tools and applications. Exp. Biol. Med. (Maywood) 235, 411-423. doi: 10.1258/ebm.2009.009230

Rejniak, K. A., Quaranta, V., and Anderson, A. R. (2012). Computational investigation of intrinsic and extrinsic mechanisms underlying the formation of carcinoma. Math. Med. Biol. 29, 67-84. doi: 10.1093/imammb/dqq021
Rejniak, K. A., Wang, S. E., Bryce, N. S., Chang, H., Parvin, B. Jourquin, J., et al. (2010). Linking changes in epithelial morphogenesis to cancer mutations using computational modeling. PLoS Comput. Biol. 6:e1000900. doi: 10.1371/journal.pcbi.1000900

Rennert, P. D., Ichimura, T., Sizing, I. D., Bailly, V., Li, Z., Rennard, R., et al. (2006). T cell, Ig domain, mucin domain-2 gene-deficient mice reveal a novel mechanism for the regulation of Th2 immune responses and airway inflammation. J. Immunol. 177, 4311-4321.

Rodriguez-Manzanet, R., DeKruyff, R. Kuchroo, V. K., and Umetsu, D. T. (2009). The costimulatory role of TIM molecules. Immunol. Rev. 229, 259-270. doi: 10.1111/j.1600065X.2009.00772.x

Salgado, J. C., Olivera-Nappa, A. Gerdtzen, Z. P., Tapia, V., Theil, E. C., Conca, C., et al. (2010). Mathematical modeling of the dynamic storage of iron in ferritin. BMC Syst. Biol. 4:147. doi 10.1186/1752-0509-4-147

Santambrogio, P., Levi, S., Cozzi, A. Rovida, E., Albertini, A., and Arosio, P. (1993). Production and characterization of recombinant heteropolymers of human ferritin $\mathrm{H}$ and L chains. J. Biol. Chem. 268, 12744-12748.

Savageau, M. A. (1972). The behavior of intact biochemical control systems. Curr. Top. Cell Regul. 6, 63-130.

Savageau, M. A. (1979). Biochemica systems analysis: a study of function and design in molecular Biology. J. Basic Microbiol. 19, 149-150. doi: 10.1016/0307-4412(77)90075-9

Savageau, M. A. (2001). Design principles for elementary gene circuits: elements, methods, and examples. Chaos 11, 142-159. doi: 10.1063/1.1349892

Thomas, S. R., and Mikulecky, D. C. (1978). A network thermodynamic model of salt and water flow across the kidney proximal tubule. Am. J. Physiol. 235, F638-F648.

Todorich, B., Zhang, X., SlagleWebb, B., Seaman, W. E., and Connor, J. R. (2008). Tim-2 is the receptor for $\mathrm{H}$-ferritin on oligodendrocytes. J. Neurochem. 107, 1495-1505. doi: 10.1111/j.14714159.2008.05678.x

Todorich, B., Zhang, X., and Connor, J. R. (2011). H-ferritin is the major source of iron for oligodendrocytes. Glia 59, 927-935. doi: 10.1002/glia.21164
Torti, S. V., and Torti, F. M. (1994). Iron and ferritin in inflammation and cancer. Adv. Inorg. Biochem. 10, 119-137.

Ulrich, C. M., Nijhout, H. F., and Reed, M. C. (2006). Mathematical modeling: epidemiology meets systems biology. Cancer Epidemiol. Biomarkers Prev. 15, 827-829. doi: 10.1158/1055-9965.EPI-06-0252

Wang, W., Di, X., D’Agostino, R. B. Jr, Torti, S. V., and Torti, F. M. (2007). Excess capacity of the iron regulatory protein system. J. Biol. Chem. 282, 24650-24659. doi: 10.1074/jbc.M703167200

Watanabe, N., Tanaka, M., Suzuki, K., Kumanogoh, A., Kikutani, H., and Miyajima, A. (2007). Tim2 is expressed in mouse fetal hepatocytes and regulates their differentiation. Hepatology 45, 1240-1249. doi: 10.1002/hep.21539

Wu, Y., Zhou, S., Qin, F., Zheng, K., and Ye, X. (2010). Modeling the oxidation kinetics of Fenton's process on the degradation of humic acid. J. Hazard. Mater. 179, 533-539. doi: 10.1016/j.jhazmat.2010.03.036

Zhu, X., Zhou, X., Lewis, M. T., Xia, L., and Wong, S. (2011). Cancer stem cell, niche and EGFR decide tumor development and treatment response: a biocomputational simulation study. J. Theor. Biol. 269, 138-149. doi: 10.1016/j.jtbi.2010.10.016

Conflict of Interest Statement: The authors declare that the research was conducted in the absence of any commercial or financial relationships that could be construed as a potential conflict of interest.

Received: 08 April 2013; accepted: 21 May 2013; published online: 07 June 2013.

Citation: Xie Z, Harrison SH, Torti SV, Torti FM and Han J (2013) Application of circuit simulation method for differential modeling of TIM-2 iron uptake and metabolism in mouse kidney cells. Front. Physiol. 4:136. doi: 10.3389/fphys. 2013.00136

This article was submitted to Frontiers in Systems Biology, a specialty of Frontiers in Physiology.

Copyright (c) 2013 Xie, Harrison, Torti, Torti and Han. This is an open-access article distributed under the terms of the Creative Commons Attribution License, which permits use, distribution and reproduction in other forums, provided the original authors and source are credited and subject to any copyright notices concerning any third-party graphics etc. 